\title{
Sideband-Enhanced Cold Atomic Source for Optical Clocks
}

\author{
Matteo Barbiero®, ${ }^{1}$ Marco G. Tarallo®, ${ }^{1,{ }^{*}}$ Davide Calonico $\odot,{ }^{1}$ Filippo Levi®, ${ }^{1}$ \\ Giacomo Lamporesi $\odot,{ }^{2}$ and Gabriele Ferrari $\oplus^{2}$ \\ ${ }^{1}$ Istituto Nazionale di Ricerca Metrologica, Strada delle Cacce 91, 10135 Turin, Italy \\ ${ }^{2}$ INO-CNR BEC Center and Dipartimento di Fisica, Università di Trento, 38123 Trento, Italy
}

(Received 12 September 2019; revised manuscript received 5 November 2019; published 9 January 2020)

We demonstrate the enhancement and optimization of a cold strontium atomic beam from a twodimensional magneto-optical trap (2D MOT) transversely loaded from a collimated atomic beam by adding a sideband frequency to the cooling laser. The parameters of the cooling and sideband beams are scanned to achieve the maximum atomic beam flux and are compared with Monte Carlo simulations. We obtain a 2.3 times larger total atomic flux and a brightness increase of a factor 4 compared with a conventional, single-frequency 2D MOT for a given total power of $200 \mathrm{~mW}$. We show that the sidebandenhanced 2D MOT can reach the loading-rate performances of space-demanding Zeeman-slower-based systems, while it can overcome systematic effects due to thermal-beam collisions and hot-black-bodyradiation shift, making it suitable for both transportable and accurate optical lattice clocks. Finally, we numerically study possible extensions of the sideband-enhanced 2D MOT to other alkaline-earth species.

DOI: 10.1103/PhysRevApplied.13.014013

\section{INTRODUCTION}

A cold, bright, and compact atomic beam source is an important asset for any experiment featuring ultracold atoms, such as atom interferometers [1], degenerate quantum gases for quantum simulation [2], and in particular, optical atomic clocks [3]. In the last case, two-valenceelectron alkaline-earth or alkaline-earth-like metals, such as $\mathrm{Ca}$ [4], $\mathrm{Sr}$ [5], $\mathrm{Mg}$ [6], and $\mathrm{Yb}$ [7], are generally used as atomic frequency discriminators and have low vapor pressures at room temperature, and thus high-temperature ovens are needed to generate enough atomic vapor, typically followed by a space-demanding Zeeman slower (ZS). Although compact and transportable versions of the "oven + ZS" atomic beam system have been developed $[8,9]$, the ZS magnetic field complicates the design of the magnetooptical trap (MOT) because of stray magnetic fields, which typically need to be compensated with extra coils [10]. Moreover, some concerns about the systematic effects due to stray magnetic fields from ZS permanent magnets [11], collisions with the atomic beam particles [12], and the hot black-body radiation (BBR) from the oven region [13] can arise below the $10^{-18}$ relative uncertainty level.

The two-dimensional- (2D) MOT atomic source $[14,15]$ can be transversely loaded, hence reducing the setup dimensions, avoiding direct exposure of the atomic reference to hot metals, and at the same time obtaining an optical shutter of the atomic beam just by turning off its

\footnotetext{
*m.tarallo@inrim.it
}

cooling beams. This avoids the use of in-vacuum mechanical shutters or optical beam deflectors [16] as is done for ZS or collimated oven beams. The 2D MOT system complexity can be further reduced by its permanent magnet implementation [17-19].

In this work, we present an alternative atomic source using a 2D MOT source of $\mathrm{Sr}$ atoms for metrological application. The mechanical implementation of the atomic source is similar to other setups built to generate lithium [17], sodium [18,20], and strontium [21] atomic beams. Our system is further characterized by a collimated atomic beam transmitted by a bundle of capillaries directly toward the 2D MOT region, and a two-frequency optical molasses to enhance the atomic flux toward the trapping region. The design, engineering, and characterization of the sideband(SB) enhanced 2D MOT strontium source is the main result of this work. This is accomplished by our looking at the loading performances of a three-dimensional (3D) MOT typically used as the first cooling and trapping stage for an optical lattice clock [22]. Monte Carlo (MC) numerical simulations are used to find the optimal optical configuration and are then compared with the experimental results.

The article is organized as follows: Sec. II introduces the physical interpretation and significance of adding a sideband frequency to the cooling beams of the 2D MOT; Sec. III describes the experimental apparatus assembled for an optical lattice clock; in Sec. IV we describe the numerical modeling of the atomic source and the 2D MOT cooling and trapping processes by Monte Carlo simulations; Sec. V 
reports the experimental characterization of our atomic source, and in Sec. VI we demonstrate how the sidebandenhancement method is able to magnify the number of trapped atoms by a magneto-optical trap. Finally, Sec. VII discusses the extension of the sideband-enhancement method to other alkaline-earth atomic species and the metrological perspectives opened by this atomic source for optical clocks.

\section{PRINCIPLES OF THE SIDEBAND-ENHANCED 2D MOT}

A 2D MOT atomic source relies on the radiationpressure friction force to capture and cool thermal atoms effusing from either an oven or a background gas. In this work, we focus on a 2D MOT loaded from a collimated atomic source, so a one-dimensional (1D) model offers good insight into the expected 2D MOT flux. For the 1D model, the number of MOT-captured atoms per second $\Phi_{2 \mathrm{D}}$ is given by [23]

$$
\Phi_{2 \mathrm{D}} \simeq n v_{\mathrm{th}} A\left(v_{c} / v_{\mathrm{th}}\right)^{4} \quad\left(v_{c} \ll v_{\mathrm{th}}\right),
$$

where $n$ is the spatial density of the thermal beam, $v_{\text {th }}=$ $\sqrt{2 k_{B} T_{\mathrm{ov}} / m}$ is the most-probable thermal velocity (for an atomic $\mathrm{Sr}$ vapor at $\left.T_{\mathrm{ov}}=460^{\circ} \mathrm{C}, v_{\mathrm{th}}=379 \mathrm{~m} / \mathrm{s}\right), A=$ $2 \pi w^{2}$ is the MOT capture surface related to the trapping beam width $w$, and $v_{c}$ is the capture velocity of the trap. It is clear from Eq. (1) that the most-influential parameter is the capture velocity $v_{c}$, which is related to the magnetic gradient $b$, the frequency detuning $\Delta$ from the cooling transition, and the total saturation parameter $s=I / I_{\text {sat }}$ of the MOT optical beams, where for an atomic transition at wavelength $\lambda$ and with spontaneous-emission rate $\Gamma$ the resonant saturation intensity $I_{\text {sat }}=\pi h c \Gamma / 3 \lambda^{3}$. In the $1 \mathrm{D}$ model, one typically computes $v_{c}$ numerically by solving the semiclassical equation of motion, as shown in Fig. 1(a). Here one can observe that there are two different dynamics inside the MOT region. In the outer region, the MOT behaves like a Zeeman slower, where the friction force exerted on any atom will be effective only if the velocity $v$ at distance $r$ from the symmetry axis is nearly resonant with the cooling laser (i.e., if the difference in the Zeeman shift and the laser detuning equals the Doppler shift). In the inner region the motion of the atoms can be described by an overdamped harmonic oscillator model. Hence, the capture velocity is strictly related to the dynamics in the outer region of the MOT and, assuming perfect compensation of the Zeeman shift and Doppler shift, it can be roughly estimated as $[17,24]$

$$
v_{c} \lesssim v_{\max }=\sqrt{a_{\max } r_{\max }},
$$

where $a_{\max }=\hbar k \Gamma / 2 m$ is the maximum acceleration at infinite saturation parameter, and $r_{\max }=\sqrt{2} w$ is the maximum interaction distance with the MOT beams, taking into account the projection of the 2D MOT beams at $45^{\circ}$ from the propagation axis of the atoms. This $v_{c}$ corresponds to the maximum velocity allowed to decelerate an atom to zero at the center of the trap. This oversimplified estimation gives us some hints on the expected performance of the 2D MOT. In particular, even for infinite available power, the capture velocity will be bounded, while the capture mechanism is fundamentally limited by the natural linewidth of the cooling transition and the cooling-beam radius. However, if one uses laser light that has several red-detuned sidebands, even faster atoms can be slowed down and the capture velocity increased [25]. Atomic beam deceleration first [26] and MOT loading enhancement afterward [27,28] were observed in alkali atomic systems by means of electro-optic modulation of the cooling beams. This technique is generally limited in power at the wavelengths of alkaline-earth atoms by electro-optic modulation because of the low-intensity damage thresholds available. Furthermore, because of the higher $\Gamma \mathrm{s}$, excessive spectral broadening would reduce the radiationpressure force, making it no longer sufficient to keep the thermal atoms in the trap. A one-sideband 3D MOT was previously realized to trap $\mathrm{Ca}$ atoms loaded directly from an effusing atomic oven [24,29]. In this case, with a total MOT saturation parameter $s \sim 0.1$ and an atomic vapor temperature of $600^{\circ} \mathrm{C}$, an enhancement factor of 7 was observed [29]. However, here only a very small fraction of the available atoms were trapped, and hence that system would be very unfavorable in the case of loading of a $2 \mathrm{D}$ MOT source.

It is more interesting to investigate the sidebandenhanced 2D MOT in the limit of high total saturation parameter $s \geq 1$, where most of the low-velocity class $\left(v \leq v_{\max }\right)$ is slowed and captured by the cooling beams. Figure 1(a) shows a simulation of the phase-space trajectories for typical values of the experimental parameters $(\Delta, s, b)$ used in a strontium 2D MOT [21]. The acceleration patterns of the sideband-enhanced 2D MOT in the atomic phase space are depicted in Fig. 1(b). As shown in the plot, the sideband beams interact with atoms from a higher-velocity class, decelerating them toward the capture region of the standard MOT beam. This increase of the capture velocity is best displayed in Fig. 1(c), where we the MOT acceleration is shown as a function of the atomic approaching velocity. In the standard MOT (dashed blue line) the force peaks around a given velocity, reaching $a_{\max }$, and the amount of power increases the spectral width of the force as $\sqrt{s}$. On the other hand, the sidebandenhanced force (dot-dashed red line) presents a second peak at higher velocity without degrading the peak acceleration. Optimal positioning of the sideband frequency thus allows an increase of the expected capture velocity $v_{c}$ and of the expected MOT loading rate too.

Another expected beneficial effect of the sidebandenhanced 2D MOT with large $s$ is the reduction of the 


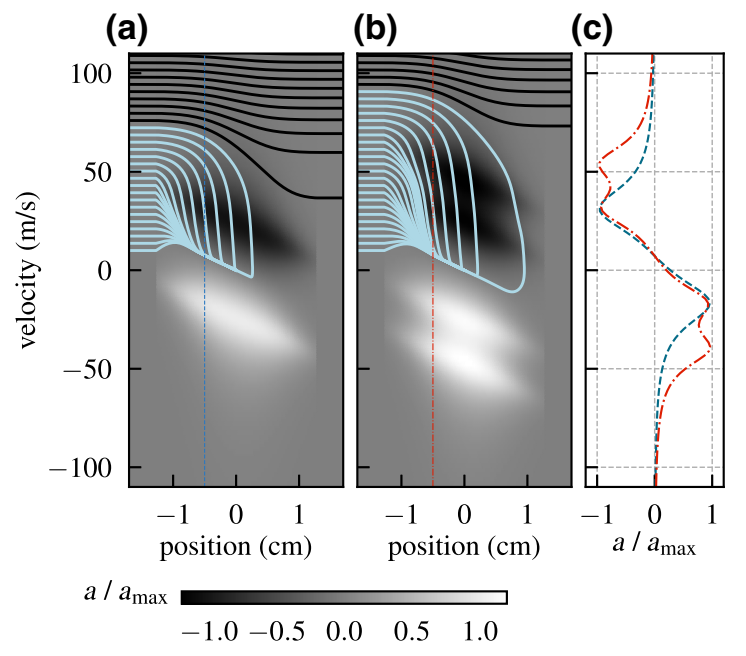

FIG. 1. One-dimensional simulation of the atomic trajectories of strontium atoms (light-blue line) for different capture processes in a 2D MOT. The color map plotted on the background depicts the acceleration value at each point of the phase space. (a) Single-frequency 2D MOT. The total saturation and the detuning of the MOT beams are $s=7$ and $\Delta / \Gamma=-1.6$, respectively. The estimated capture velocity is $72(1) \mathrm{m} / \mathrm{s}$. (b) Sidebandenhanced 2D MOT. The saturation parameter is $s=3.5$ for the 2D MOT beam with $\Delta / \Gamma=-1.6$ and $s_{\mathrm{SB}}=3.5$ for the sideband beam with $\Delta_{\mathrm{SB}} / \Gamma=-3.2$. The estimated capture velocity is $90(1) \mathrm{m} / \mathrm{s}$. The magnetic field gradient and beam width used in this calculation are $b=0.22 \mathrm{~T} / \mathrm{m}$ and $w=1 \mathrm{~cm}$, respectively. (c) Acceleration profile at $r=-w / 2$ of the sideband trapping (red line) and standard 2D MOT trapping (blue line).

transverse temperature of the cold atomic sample compared with the standard 2D MOT, which would yield a higher brightness (i.e., lower beam divergence). This can be explained considering that the optical power redistributed at a higher frequency weakly interacts with the atoms trapped once they reach the center of the MOT.

To correctly address the expected performances of a sideband-enhanced strontium 2D MOT, we perform a dedicated MC simulation that takes into account the actual geometry of the system, the magnetic field gradient, the residual divergence of the atomic beam from the oven, and the expected loading rate for the final 3D MOT. This is described in detail in Sec. IV.

\section{EXPERIMENTAL APPARATUS}

\section{A. Vacuum system}

A schematic drawing of the vacuum system used to produce and trap ultracold strontium atoms is depicted in Fig. 2. It was described in Ref. [30], and its concept is adapted from previous work $[17,18]$. The vacuum system is conceived to host two physical regions with very different vacuum levels, the atomic source region and the science-cell region, and at the same time to be

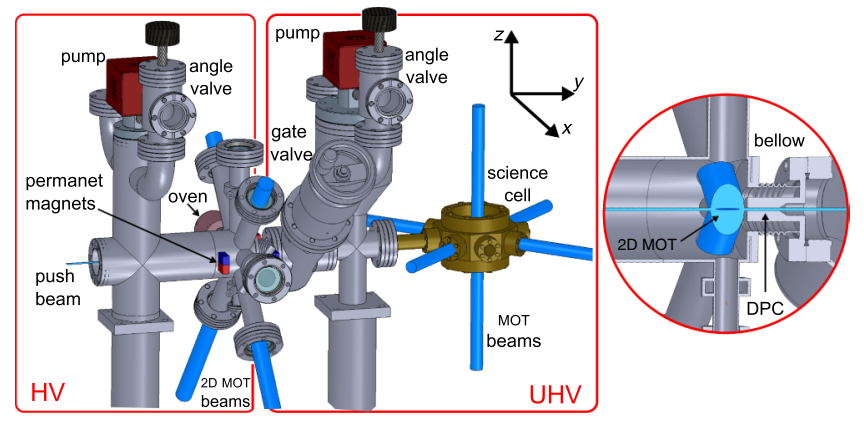

FIG. 2. The vacuum system. It hosts a high-vacuum (HV) region for atomic beam production and an ultrahigh-vacuum (UHV) region for cooling and trapping the atomic sample. A DPC connects the two regions. The size of the entire vacuum apparatus is roughly $70 \times 70 \times 45 \mathrm{~cm}^{3}$.

very compact. The atomic source region consists of a stainless-steel vacuum chamber with a multiway cross at its end, where the intersection plane of the tubes forms the 2D MOT plane. The ultrahigh-vacuum region hosts a small octagonal science cell with two large vertical optical accesses (DN63CF) and seven small lateral optical windows (DN16CF) for cooling, trapping, and operating a Sr optical clock. The two vacuum regions are connected by a differential pumping channel (DPC) carved in a custom bellow with 2-mm diameter and 22.8-mm length and an all-metal gate valve. The DPC sets the maximum divergence of the cold atomic beam as $87 \mathrm{mrad}$, while a conductance of $4.3 \times 10^{-2} \mathrm{l} / \mathrm{s}$ allows us to maintain a differential pressure of $10^{4}$ between the two regions. Vacuum is maintained by two ion-getter pumps with a pumping speed for hydrogen of 200 and $500 \mathrm{l} / \mathrm{s}$, respectively. Both regions reach a background pressure below $10^{-10} \mathrm{mbar}$ when the oven is not heated, while the pressure in the ultrahigh-vacuum region remains less than the minimum detectable value when the oven is hot.

\section{B. Collimated atomic source}

The oven consists of a simple stainless-steel cylinder with an aperture of $16 \mathrm{~mm}$ and a conflat flange (DN16CF) for attachment to the main body of the vacuum system on one of its circular sides. The oven is attached to the multiway-cross vacuum chamber $128 \mathrm{~mm}$ from its center. To produce a collimated atomic beam, an array of $N_{\text {cap }} \simeq 150$ capillaries made of nickel-based alloy (Monel 400), with internal radius $r_{\text {cap }}=0.2 \mathrm{~mm}$ and length $L_{\text {cap }}=20 \mathrm{~mm}$, is inserted at the oven aperture. The capillaries are tightened inside a holder that lies in the aperture of the oven. Heating is ensured by a pair of heating cartridges. The $\mathrm{Sr}$ vapor is typically generated at temperature $T_{\mathrm{ov}}=460^{\circ} \mathrm{C}$. To avoid clogging of the capillaries with strontium, the oven hosts an extra pair of heating cartridges close to its aperture to 
maintain the capillaries at a temperature $T_{\text {cap }}$ higher than $T_{\mathrm{ov}}$. For all experimental characterizations, we maintain a differential temperature $T_{\text {cap }}-T_{\mathrm{ov}}=30^{\circ} \mathrm{C}$. At the typical operational oven temperature $T_{\mathrm{ov}}=460^{\circ} \mathrm{C}$, the estimated vapor pressure inside is $p_{\mathrm{ov}}=0.133 \mathrm{~Pa}$ [31], from which we estimate the $\mathrm{Sr}$ atomic density by means of the ideal-gas law: $n_{\mathrm{ov}}=p_{\mathrm{ov}} / k_{B} T_{\mathrm{ov}}=1.31 \times 10^{19}$ atoms $/ \mathrm{m}^{3}$. In the regime of negligible collisions inside the capillaries [mean free path $\lambda_{\text {ov }}=\left(\sqrt{2} n_{\mathrm{ov}} \sigma_{\mathrm{Sr}}\right)^{-1} \sim 70 \mathrm{~mm} \gg L_{\text {cap }}$, with $\sigma_{\mathrm{Sr}}=8 \times 10^{-19} \mathrm{~m}^{2}$ the elastic cross section], the atomic flux is proportional to the oven pressure $p_{\mathrm{ov}}$ and is estimated as [32]

$$
\Phi_{\mathrm{ov}}=\tilde{a} \frac{4 \sqrt{\pi}}{3} \frac{n_{\mathrm{ov}} v_{\mathrm{th}} r_{\mathrm{cap}}^{3}}{L_{\mathrm{cap}}} N_{\mathrm{cap}}
$$

where $\tilde{a}$ is the isotopic abundance. In the case of ${ }^{88} \mathrm{Sr}$, the expected atomic flux $\Phi_{\mathrm{ov}}$ at $T_{\mathrm{ov}}=460^{\circ} \mathrm{C}$ is $5.8 \times 10^{14}$ atoms $/ \mathrm{s}$. The geometrical constraint imposed by the capillaries yields a theoretical divergence angle $\theta_{\text {cap }} \simeq r_{\text {cap }} / L_{\text {cap }}=20 \mathrm{mrad}$.

\section{2D MOT and cold-atomic-source generation}

As sketched in Fig. 2, the 2D MOT is composed of a 2D quadrupole magnetic field in combination with two orthogonal pairs of retroreflected laser beams of opposite circular polarization.

The magnetic field gradient is generated by four stacks of permanent magnets [17]. Each stack is composed of nine neodymium bar magnets with size of $25 \times 10$ $\times 3 \mathrm{~mm}^{3}$ and magnetization of $6.6(1) \times 10^{5} \mathrm{~A} / \mathrm{m}$. The stacks are placed around the center of the 2D MOT at the positions $\mathbf{r}_{m}= \pm \mathbf{x}_{0}, \pm \mathbf{y}_{0}$, where $x_{0}=110 \mathrm{~mm}$ and $y_{0}=90 \mathrm{~mm}$. The magnetization of each permanent magnet is oriented in such a way that it has the same direction with its neighbor along the $y$ axis and opposite direction with its neighbor along the $x$ axis. We estimate the generated field on the 2D MOT plane by finite-element analysis (FEA). This shows a uniform linear gradient $\mathbf{B}_{m}(\mathbf{r})=$ $b \mathbf{x}-b \mathbf{z}$ close to the center of the trap $|\mathbf{r}|<1 \mathrm{~cm}$ with $b=0.224 \mathrm{~T} / \mathrm{m}$. The maximum deviation between the linear approximation $\mathbf{B}_{m}(\mathbf{r})$ and the FEA estimation is negligible within the 2D MOT trapping volume, as it ultimately amounts to $\Delta_{Z}=2 \pi \times 5.6 \mathrm{MHz}=0.17 \Gamma$ in frequency detuning.

The two pairs of counterpropagating beams allow magneto-optical cooling and trapping of slow atoms effusing from the oven along the $x$ and $z$ axes, while they are free to drift along the $y$ axis. Hence, a nearly resonant laser "push" beam is directed to the 2D MOT center along the $y$ axis toward the ultrahigh-vacuum science cell to launch atoms collected in the 2D MOT toward the MOT region. The center of the MOT in the science cell is located 370 $\mathrm{mm}$ from the 2D MOT center. Finally, the mandatory MOT quadrupole field is generated by a pair of coils with the current flowing in the anti-Helmholtz configuration, which generates a typical magnetic field gradient of $0.4 \mathrm{~T} / \mathrm{m}$.

\section{Laser system}

The 461-nm laser light is provided by a semiconductorbased commercial laser composed of an infrared master laser, a tapered amplifier, and a second-harmonicgeneration cavity. It is able to generate up to $600 \mathrm{~mW}$ of blue power. This blue laser light is split into six main optical paths and frequency manipulated by acoustooptic modulators (AOMs). The laser frequency is stabilized to the $\mathrm{Sr}^{1} S_{0}-{ }^{1} P_{1}$ atomic transition by performing wavelength-modulation saturation spectroscopy on a hot vapor of strontium generated in a heatpipe [33]. Typically, we are able to deliver about half of the available power to the atoms.

A detailed scheme of the various beam paths is depicted in Fig. 3. For typical experimental conditions, the 2D MOT and sideband beams share $200 \mathrm{~mW}$ and have a $1 / e^{2}$ beam width $w_{2 \mathrm{D}}=9.5 \mathrm{~mm}$, the MOT beams have a total power of $45 \mathrm{~mW}$, a width of $6.2 \mathrm{~mm}$, and a detuning from the atomic resonance of $-1.2 \Gamma$, the push beam has a power of up to $5 \mathrm{~mW}$ and a width of $0.81 \mathrm{~mm}$, the spectroscopy

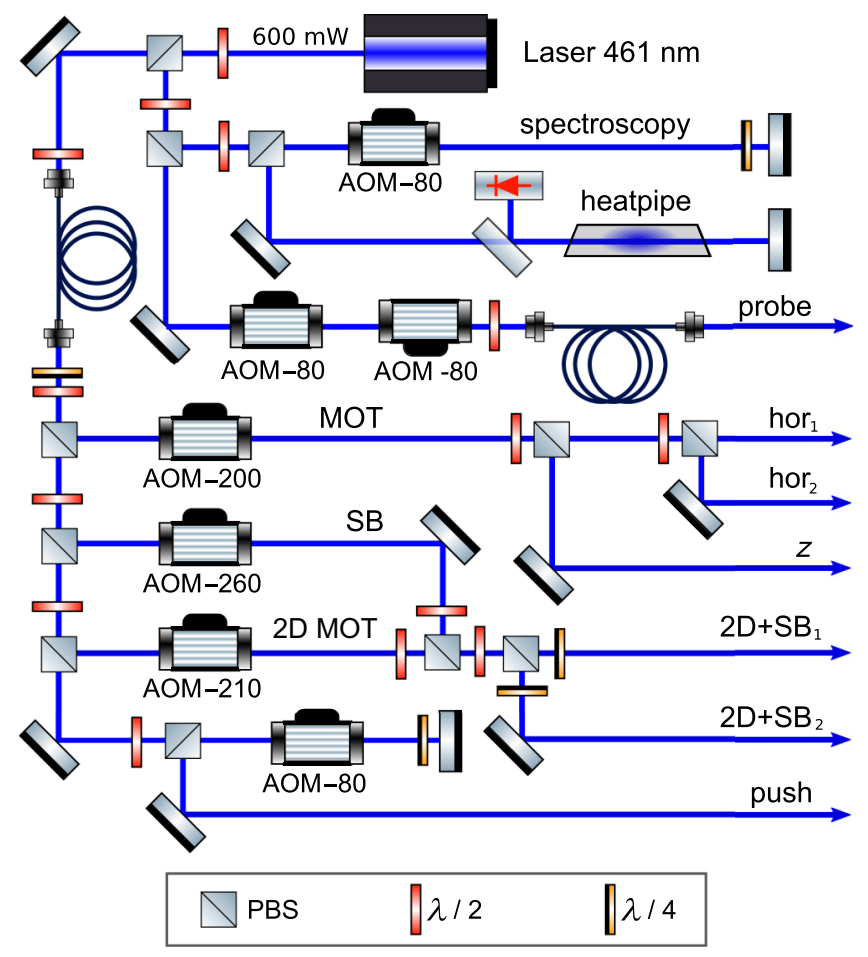

FIG. 3. Optical setup of the blue-laser system used for cooling, trapping, and probing ultracold strontium atoms. Each AOM is drawn with its driving frequency (in megahertz), and the sign corresponds to the diffraction order. Beam-shaping lenses are not shown. PBS, polarizing beam splitter. hor ${ }_{1}$ and hor ${ }_{2}$ are the two orthogonal MOT beams in the $x-y$ plane, respectively. 
beam sent inside the heatpipe has a power of $0.5 \mathrm{~mW}$ and a width of $0.37 \mathrm{~mm}$, and finally the probe beam has a power of $0.5 \mathrm{~mW}$ and a width of $0.83 \mathrm{~mm}$. The detuning from the atomic resonance of the beams used in the atomic source system (2D MOT, sideband, and push beams) is scanned for optimal atomic flux, as described in Sec. V.

We generate the 2D MOT main and sideband beams as follows: two dedicated 200- and 350-MHz AOMs are used to shift the frequencies of two beams, which are shaped with the same telescope in order to have the same beam width. They are combined in a polarizing-beam-splitter cube with orthogonal linear polarizations in such a way that the 2D MOT (sideband) beam is completely transmitted (reflected). The two-beam polarization is then rotated $45^{\circ}$ by a half-wavelength retarding waveplate, and thus the two beams are recombined in a second polarizing beam splitter, which yields the two beams for the branches of the 2D optical molasses. The $350-\mathrm{MHz}$ AOM is dedicated to the sideband beam, offering a bandwidth of $100 \mathrm{MHz}$ to find the optimal frequency that maximizes the loading of the atomic source.

\section{NUMERICAL SIMULATION OF THE 2D MOT}

$\mathrm{MC}$ simulation is a powerful and versatile numerical approach because it allows one to study complex physical processes in a realistic environment: from the simple MOT capture process [34-37], to the loading process of an optical potential [38,39], a molecular MOT [40], and a Rydberg-dressed MOT [41]. Knowing the atom-light interactions and the geometry of the system, we want to extract the capture efficiency of our 2D MOT system for a given trapping configuration, defined by the 2D MOT beams, sideband beams, and push beam, as described in the previous section. The MC algorithm is implemented in PYTHON.

We simulate $N_{\text {sim }}=2 \times 10^{4}$ trajectories of atoms that interact with the trap. At initial time $t=0$, the starting positions of atoms are randomly sampled in a disk region of radius $r_{0}=7.5 \mathrm{~mm}$ in the $y-z$ plane and at $x_{0}=$ $-128 \mathrm{~mm}$ far from 2D MOT trap center along the direction of hot atomic flux emitted by the oven. The velocity space is sampled from the Maxwell-Boltzmann probability distribution expressed in polar coordinates. The sampling of the absolute value of the starting atomic velocity $v_{0}$ is limited to $v_{\text {cut }}=90 \mathrm{~m} / \mathrm{s}$ to speed up the calculation. The polar angle $\theta_{0}$ is uniformly sampled considering the geometrical constraint imposed by the capillaries $\theta_{0} \leq \theta_{\text {cap }}$. The azimuthal angle $\phi_{0}$ is randomly chosen between 0 and $2 \pi$.

The trajectory is discretized in time with step size $\delta t=50 \mu \mathrm{s}$, and is computed until $t_{\text {tot }}=4 \mathrm{~ms}$ by use of a Runge-Kutta algorithm [42]. The time step $\delta t$ is chosen to be greater than the internal atomic timescale $\tau_{P_{1}}=\Gamma^{-1}$ so that the atom-light interaction can be calculated by use of the semiclassical approximation of the optical Bloch equations but less than the capture time $\Delta t_{\max }$ for an atom moving at $v_{\max }$, which is about $165 \mu \mathrm{s}$. At each time step $t_{i}=i \delta t$, the atom-light scattering rate with a single laser beam is computed as

$$
R\left(t_{i}\right)=\frac{\Gamma}{2} \frac{s\left[\mathbf{r}\left(t_{i}\right)\right]}{1+s\left[\mathbf{r}\left(t_{i}\right)\right]+4\left(\frac{\Delta_{\mathrm{eff}}\left[\mathbf{r}\left(t_{i}\right), \mathbf{v}\left(t_{i}\right)\right]}{\Gamma}\right)^{2}},
$$

where $s\left[\mathbf{r}\left(t_{i}\right)\right]$ is the position-dependent saturation parameter and $\Delta\left[\mathbf{r}\left(t_{i}\right), \mathbf{v}\left(t_{i}\right)\right]$ is the frequency detuning due to the Doppler and Zeeman shift. The local saturation parameter is computed as

$$
s\left[\mathbf{r}\left(t_{i}\right)\right]=s_{0} \exp \left(-\frac{2\left|\mathbf{r}\left(t_{i}\right) \times \hat{\mathbf{k}}\right|^{2}}{w^{2}}\right),
$$

where $s_{0}$ is the saturation peak, $w$ is the width of the optical beam, and the vector product $\mathbf{r} \times \hat{\mathbf{k}}$ is the distance between the atom position and the center of the laser-line propagation described by the unit vector $\hat{\mathbf{k}}$. Considering the aperture of the optical elements, a spatial cutoff of $|\mathbf{r} \times \hat{\mathbf{k}}|<1.2 \mathrm{~cm}$ in the local saturation parameter is also applied. The frequency detuning is computed as

$$
\Delta_{\text {eff }}\left[\mathbf{r}\left(t_{i}\right), \mathbf{v}\left(t_{i}\right)\right]=\Delta+\mathbf{k} \cdot \mathbf{v}\left(t_{i}\right)-\frac{\mu_{B}}{\hbar}\left|\mathbf{B}\left[\mathbf{r}\left(t_{i}\right)\right]\right|,
$$

where $\Delta$ is the laser-frequency detuning from the atomic transition, $\mathbf{k} \cdot \mathbf{v}\left(t_{i}\right)$ is the Doppler shift, and the last term is the Zeeman shift induced by the atomic position in the magnetic field $\mathbf{B}\left[\mathbf{r}\left(t_{i}\right)\right]$ described in Sec. III.

The heating induced by the spontaneous-emission process is also taken into consideration in the simulated dynamics by our adding a random recoil momentum $\hbar|\mathbf{k}| \sqrt{R \delta t} \hat{\mathbf{e}}$, where $R \delta t$ is the average number of scattering events in time interval $\delta t$, while $\hat{\mathbf{e}}$ is a unit vector randomly chosen from an isotropic distribution [35]. The resulting atom's acceleration induced by the 2D MOT (and sideband) beams is described according to

$$
\mathbf{a}_{2 \mathrm{D}, \mathrm{SB}}=\frac{\hbar|\mathbf{k}|}{m} \sum_{n=0}^{4} \frac{R_{n}}{4}\left(\hat{\mathbf{k}}_{n}+\frac{\hat{\mathbf{e}}_{n}}{\sqrt{R_{n} \delta t / 4}}\right),
$$

where the saturation peak $s_{2 \mathrm{D}, \mathrm{SB}}$ is redistributed equally among the four beams of the 2D MOT and sideband and the beam directions $\hat{\mathbf{k}}_{n}$ are described by the four combinations of the unit vectors $( \pm \hat{\mathbf{x}} \pm \hat{\mathbf{z}}) / \sqrt{2}$. The acceleration induced by the push beam is computed as

$$
\mathbf{a}_{\text {push }}=\frac{\hbar|\mathbf{k}|}{m} R_{\text {push }}\left(\hat{\mathbf{y}}+\frac{\hat{\mathbf{e}}}{\sqrt{R_{\text {push }} \delta t}}\right) .
$$

The total acceleration $\mathbf{a}\left(t_{i}\right)$ exerted on the atom at position $\mathbf{r}\left(t_{i}\right)$ with velocity $\mathbf{v}\left(t_{i}\right)$ is quantified as the sum of the 
above processes:

$$
\mathbf{a}=\mathbf{a}_{2 \mathrm{D}}+\mathbf{a}_{\mathrm{SB}}+\mathbf{a}_{\text {push }} .
$$

Once $t=t_{\text {tot }}$, each simulated atom is considered captured in the MOT if the divergence of the atomic trajectory computed along the push direction is lower that the geometrical constraint imposed by the MOT capture angle $\theta_{\text {MOT }}=16 \mathrm{mrad}$ and if the final longitudinal velocity is below the MOT capture velocity $v_{\text {capt }}^{\mathrm{MC}}=60 \mathrm{~m} / \mathrm{s}$. In the selection of the captured trajectories, we also consider the losses due to collisions with hot atoms from the thermal beam, whose timescale $\tau_{\text {coll }}$ is calculated to be $50 \mathrm{~ms}$. Hence, for each trajectory the collision probability $p_{\text {coll }}$ is estimated as $1-e^{-\tau_{2 \mathrm{D}} / \tau_{\text {coll }}}$ and a unitary random number $\varepsilon$ is generated to accept $\left(\varepsilon>p_{\text {coll }}\right)$ or reject $(\varepsilon<$ $\left.p_{\text {coll }}\right)$ each simulated atomic trajectory. Figure 4 shows the results from two simulation runs, where the final transverse velocity and position coordinates are displayed versus the number of occurrences. The resulting velocity distribution is used to estimate the transverse temperature of the atomic beam.

Finally, the capture-efficiency ratio $r$ is defined as $r=N_{\text {tr }} / N_{\text {sim }}$, where $N_{\text {tr }}$ is the number of captured trajectories for a given trapping configuration. Besides a scaling factor, $r$ is used as a comparison with the experimental data. The numerical results for such a modeled atomic source are presented in Secs. V and VI together with the experimental data.
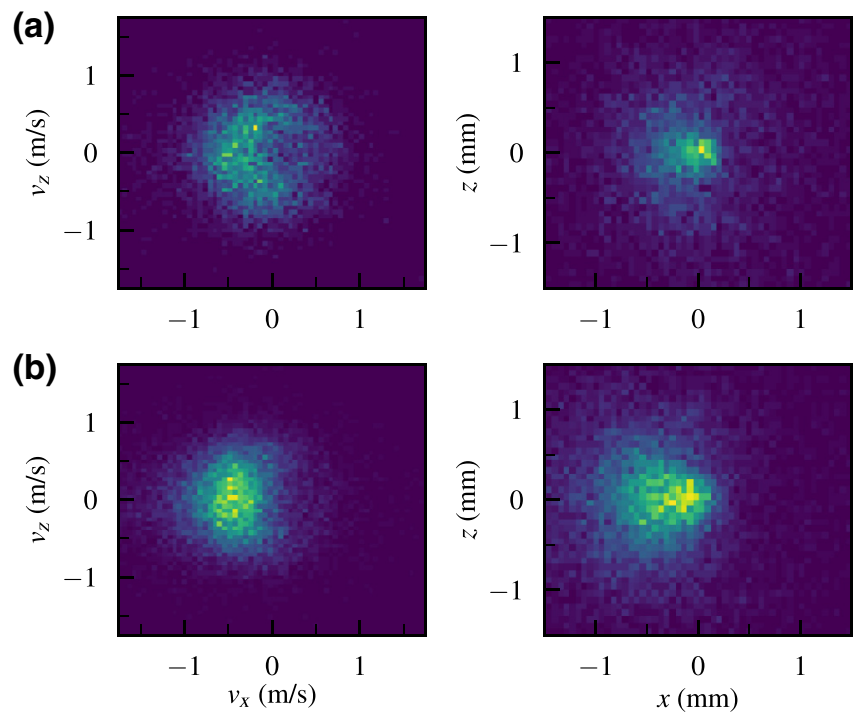

FIG. 4. Monte Carlo simulation of the velocity and position transverse coordinates of the 2D MOT-generated atomic beam at the end of the simulation time $t_{\text {tot }}$. Density maps of the captured trajectories for (a) the single-frequency 2D MOT $\left(s_{2 \mathrm{D}}=s_{\text {tot }}\right.$ $\left.=6.56, \Delta_{2 \mathrm{D}}=-1.6 \Gamma\right)$ and $(\mathrm{b})$ adding a sideband beam $\left(s_{\mathrm{SB}}\right.$ $\left.=3.45, s_{2 \mathrm{D}}=s_{\mathrm{tot}}-s_{\mathrm{SB}}, \Delta_{\mathrm{SB}}=-3.1 \Gamma\right)$.

\section{ATOMIC SOURCE CHARACTERIZATION}

In Fig. 5 we report the results of the characterization of our strontium 2D MOT atomic source. These are obtained by our looking at the loading of the MOT in the science chamber. Laser parameters of the 2D MOT and push beams, $\Delta_{2 \mathrm{D}}, s_{2 \mathrm{D}}$, and $\Delta_{\text {push }}$, are scanned for optimal settings to find the maximum loading rate (blue points) and are then compared with the expected capture ratio $r$ from $\mathrm{MC}$ simulation (turquoise region).

Regarding the 2D MOT beam parameters, Fig. 5(a) shows the number of atoms loaded in the MOT reaches the maximum value at $\Delta_{2 \mathrm{D}}=-1.6 \Gamma$, with an FWHM on the order of $1.5 \Gamma$. The peak position and the spectral response are in good agreement with the simulated ones. Figure 5(b) shows the increase of the number of atoms in the MOT as a function of the 2D MOT optical intensity at $\Delta_{2 \mathrm{D}}=-1.6 \Gamma$. Here we can observe that for $s_{2 \mathrm{D}}>6$, the number of atoms in the MOT starts to saturate, but much later than the unity value. The same result is predicted by the MC simulation.

We observe an optimal push intensity around $s_{\text {push }} \simeq$ 0.34 ; beyond this value the MOT number of atoms decreases, as previously verified in a similar setup [21]. The reduced efficiency in the transfer from the 2D MOT to the blue MOT is explained considering that atoms accelerated beyond $v_{\text {capt }}$ cannot be captured in the MOT. This behavior at higher $s_{\text {push }}$ is also observed in the MC simulation considering only the atoms captured in the MOT at longitudinal $v_{L}$ velocity below $v_{\text {capt }}^{\mathrm{MC}} \sim 60 \mathrm{~m} / \mathrm{s}$. Figure 5(c) shows the MOT number of atoms as a function of the pushbeam detuning. From this plot we observe that the greatest transfer efficiency is obtained near the atomic resonance $\Delta_{\text {push }}=0$, but it is not a critical parameter.

At the best trapping configuration the total atomic flux generated by the 2D MOT source is measured by our detecting the fluorescence generated by a probe beam sent along the $z$ direction, nearly at the center of the MOT in the science chamber. The resulting atomic flux $\Phi_{2 \mathrm{D}}$ reaches a maximum of $6(1) \times 10^{8}$ atoms/s, as shown in Fig. 6 . This can be compared with the MOT loading rate $L_{\mathrm{MOT}}$ and with the expected flow resulting from the capture-efficiency ratio from $\mathrm{MC}$ simulations. The MOT loading rate is given simply by $L_{\mathrm{MOT}}=N_{0} / \tau=3.1(4) \times 10^{8}$ atoms $/ \mathrm{s}$, where $\tau$ is the MOT relaxation time, which in our system without repumping is $17(2) \mathrm{ms}$ and $N_{0}=5.3(2) \times 10^{6}$ atoms is the maximum number of atoms trapped in the final MOT. It corresponds to roughly $51 \%$ of the total flux. The expected atomic flux generated by the 2D MOT can be estimated as

$$
\Phi_{2 \mathrm{D}}^{(\mathrm{th})}=r f_{\text {cut }} p_{\mathrm{rad}} \Phi_{\mathrm{ov}}=1.5 \times 10^{9} \text { atoms } / \mathrm{s} .
$$

In this estimate we use $r=6.9 \times 10^{-2}$ from MC results, $f_{\text {cut }}=1.53 \times 10^{-3}$ is the fraction of simulated velocities from the Maxwell-Boltzmann distribution considering a 
(a)

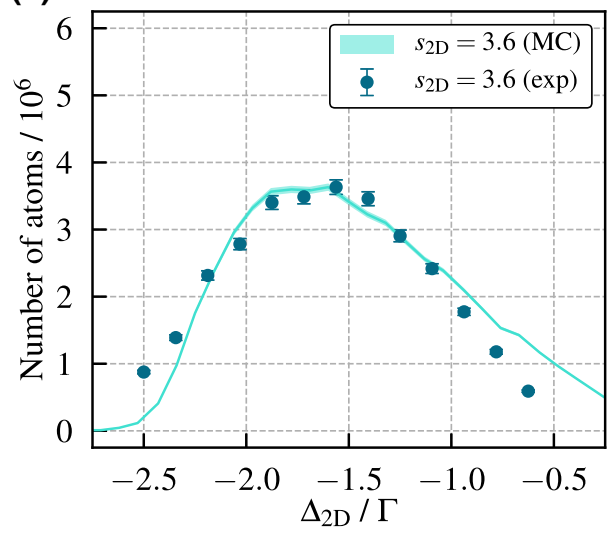

(b)

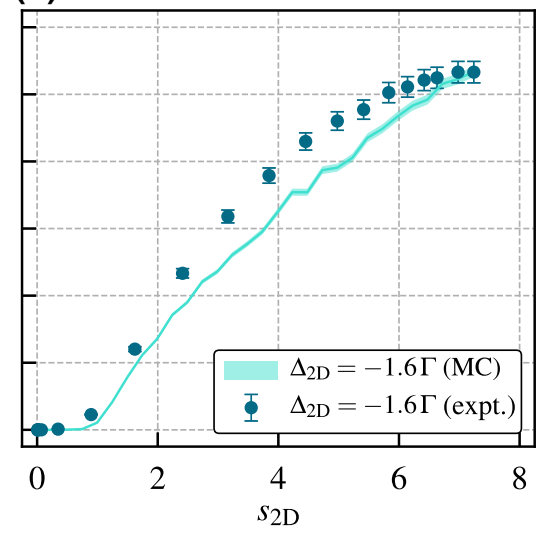

(c)

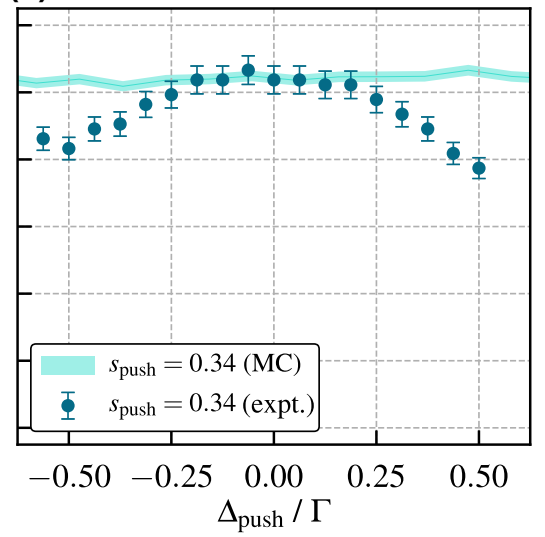

FIG. 5. Experimental characterization of the strontium atomic source in the single-frequency 2D MOT configuration. (a) Number of atoms loaded in the MOT as a function of the 2D MOT detuning $\Delta_{2 \mathrm{D}}$ at fixed saturation parameter $s_{2 \mathrm{D}}=3.6$. The blue points are the experimental values of the number of atoms in the MOT. The turquoise regions are the MC simulations properly scaled with the experimental points. (b) Number of atoms in the MOT as function of the 2D MOT saturation $s_{2 \mathrm{D}}$ at fixed detuning $\Delta_{2 \mathrm{D}}=-1.6 \Gamma$. (c) Number of atoms in the MOT as a function of the push detuning $\Delta_{\text {push }}$ at fixed saturation $s_{\text {push }}=0.34$. All the data are taken at $T_{\mathrm{ov}}=460^{\circ} \mathrm{C}$.

cutoff at $v_{\text {cut }}=90 \mathrm{~m} / \mathrm{s}$, and $p_{\text {rad }}$ is the survival probability from optical pumping to the metastable ${ }^{3} P_{2}$ state [22] that we calculated considering a typical time spent in the 2D MOT region, $\left\langle\tau_{2 \mathrm{D}}\right\rangle=2.9(6) \mathrm{ms}$, and pumping rate $R=223 \mathrm{~Hz}$, which give $p_{\text {rad }} \simeq 1-R\left\langle\tau_{2 \mathrm{D}}\right\rangle=0.35$. The estimated theoretical flux $\Phi_{2 \mathrm{D}}^{\text {(th) }}$ provides a discrepancy from the measured $\Phi_{2 \mathrm{D}}$ of only a factor of 2.4, which is remarkably close. Our simulations do not consider effects due to experimental imperfections, such as the misalignment of the zero magnetic field of the permanent magnets and the optimal push-beam direction for optical transfer to the MOT in the science chamber.

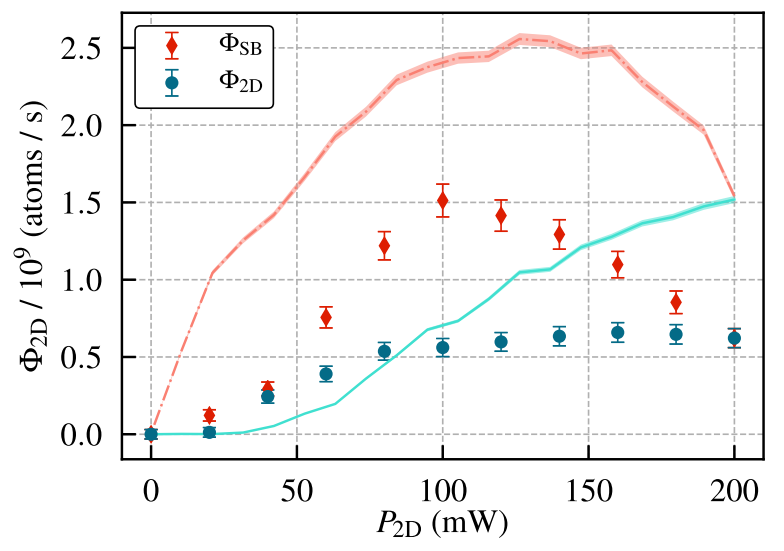

FIG. 6. Atomic flux generated from the atomic source as a function of the 2D MOT saturation parameter, without (blue circles) and with (red diamonds) the use of the sideband $\left(P_{\mathrm{SB}}=200 \mathrm{~mW}-P_{2 \mathrm{D}}\right)$. These data are taken at $s_{\text {push }}=0.34$, $\Delta_{2 \mathrm{D}} / \Gamma=-1.6, \Delta_{\mathrm{SB}} / \Gamma=-3.13$ and compared with MC estimates (shaded lines).

\section{SIDEBAND ENHANCEMENT}

\section{A. Loading a MOT with sideband enhancement}

We demonstrate sideband-enhanced loading of a 2D MOT atomic source by overlapping a second laser beam with higher-frequency detuning with the 2D MOT cooling lasers, as described in Sec. III. Figure 7 shows how the power distribution between the two frequencies affects the number of atoms collected in the MOT trap at sideband detuning $\Delta_{\mathrm{SB}}=-3.13 \Gamma$, while the $2 \mathrm{D}$ MOT beam is tuned at its previously shown maximum $\Delta_{2 \mathrm{D}}=-1.6 \Gamma$. From Fig. 7 we see that there is an optimal power distribution around $s_{\mathrm{SB}} \simeq 3.5$ that maximizes the number of atoms trapped in the MOT, reaching up to $1.2 \times 10^{7}$ atoms; that is, about 2.3 times higher than with the total available power sent to the 2D MOT AOM and about 4 times higher than the corresponding value with the sideband beam blocked.

To find the optimal working point of the sidebandenhanced 2D MOT, we scan over the sideband AOM frequency from 230 to $335 \mathrm{MHz}$, which corresponds to a detuning range between $-5 \Gamma$ and $-2.2 \Gamma$, and measure the MOT trapped atoms $N$ at different sideband powers. We perform this scan with total power $P_{\text {tot }}=200$ and 110 $\mathrm{mW}$; that is, total saturation parameter $s_{\text {tot }}=6.56$ and 3.61, respectively. We introduce the enhancement parameter $\eta$ as

$$
\eta\left(s_{\mathrm{SB}}, \Delta_{\mathrm{SB}}\right)=\frac{N\left(s_{\mathrm{SB}}=s_{\mathrm{tot}}-s_{2 \mathrm{D}}, \Delta_{\mathrm{SB}}\right)}{N\left(s_{2 \mathrm{D}}=s_{\mathrm{tot}}\right)} .
$$

The so-defined parameter $\eta$ compares the two different trapping configurations, both sharing the same total optical power $s_{\text {tot }}$. When $\eta>1$, sideband enhancement is achieved. 


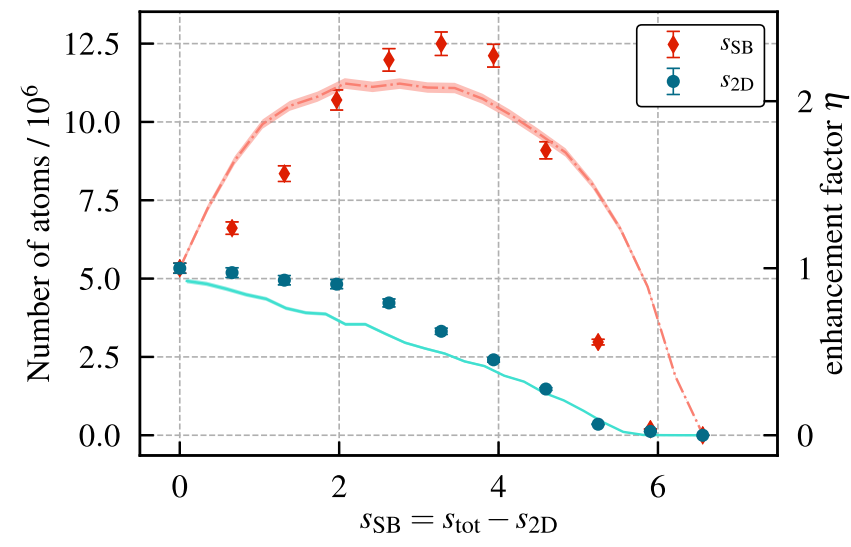

FIG. 7. Number of atoms captured in the MOT region for different power distributions of $s_{\text {tot }}=6.56$ between the sideband beam $s_{\mathrm{SB}}$ and the $2 \mathrm{D}$ MOT beam $s_{2 \mathrm{D}}$. All the data are measured with $2 \mathrm{D}$ MOT detuning $\Delta_{2 \mathrm{D}}=-1.6 \Gamma$ and sideband detuning $\Delta_{\mathrm{SB}}=-3.13 \Gamma$. The red diamonds are the number of atoms trapped in the MOT at increasing sideband-beam saturation parameter $s_{\mathrm{SB}}$. The pink shaded area represents the $\mathrm{MC}$ simulation in this experimental configuration. The blue circles describe the corresponding number of atoms trapped in the MOT with the sideband-beam AOM turned off.

Figures $8(\mathrm{a})$ and $8(\mathrm{c})$ show two sets of the sidebandenhancement-parameter scan, where we plot the enhancement parameter $(\eta)$ with respect to $s_{\mathrm{SB}}$ and $\Delta_{\mathrm{SB}}$ when $P_{\text {tot }}=200$ and $110 \mathrm{~mW}$, respectively. These results are compared with the respective MC simulations [Figs. 8(b) and $8(d)]$. The data show that the optimum loading efficiency of the final MOT is reached when the sideband frequency is tuned to $\Delta_{\mathrm{SB}}=-3.13 \Gamma$ for both totalpower regimes. At $P_{\text {tot }}=200 \mathrm{~mW}$, we reach a maximum enhancement of $\eta^{\exp }=2.3(1)$ when $s_{\mathrm{SB}} \simeq 3.1\left(P_{\mathrm{SB}} \simeq\right.$ $90 \mathrm{~mW})$. The $\mathrm{MC}$ numerical data present essentially the same main features as the experimental measurements, both having the maximum loading at the same sidebandparameter point, reaching a slightly lower enhancement $\eta^{\mathrm{MC}}=2.13(4)$, as detailed in Fig. 7. At $P_{\text {tot }}=110 \mathrm{~mW}$, we obtain the greatest enhancement factor of $\eta^{\exp }=$ $1.48(7)$ when $s_{\mathrm{SB}}=1.3\left(P_{\mathrm{SB}} \simeq 40 \mathrm{~mW}\right)$, while the $\mathrm{MC}$ numerical results show a slightly higher enhancement of $\eta^{\mathrm{MC}}=1.87(3)$. Both the numerical data and the experimental data suggest that for $s_{\text {tot }} \geq 1$, sideband enhancement increases with the increasing available power, where the optimized power distribution can be more effective. Alternatively, the rate at which the atomic flux increases with respect to the laser power is significantly higher for the case where we add the higher-detuned frequency sideband.

At maximum $\eta^{\exp }=2.3(1)$, we measure $N=1.25(4) \times$ $10^{7}$ atoms trapped in the MOT, which corresponds to a loading rate $L_{\mathrm{MOT}}^{\mathrm{SB}}$ of $7.3(9) \times 10^{8}$ atoms/s. A total flux measurement by fluorescence detection is also performed for the sideband-enhanced 2D MOT and is reported in Fig. 6. In this case we measure an atomic flux $\Phi_{\mathrm{SB}}$ of $1.5(2) \times 10^{9}$ atoms $/ \mathrm{s}$. The related enhancement factor is 2.4(4), and it is in agreement with the experimental and numerical results reported for the MOT loading.

Compared with other $\mathrm{Sr}$ atomic sources, our sideband-enhanced 2D MOT source shows high transfer
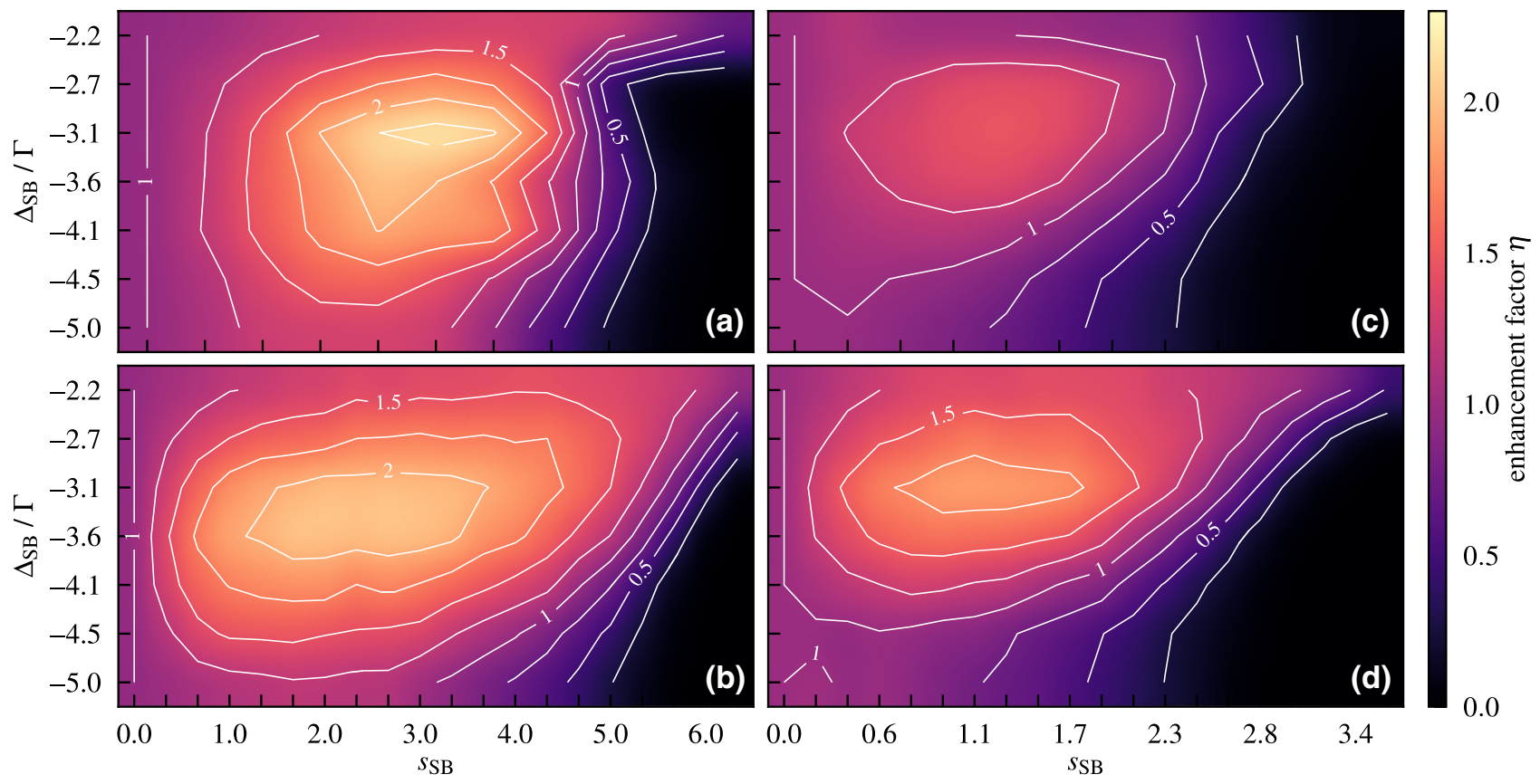

FIG. 8. Enhancement factor $\eta$ as a function of the sideband-parameter scan. (a) Experimental data compared with (b) MC numerical results at fixed total saturation parameter $s_{\text {tot }}=6.56$ and $\Delta_{2 \mathrm{D}}=-1.6 \Gamma$. (c),(d) Same as (a),(b) but for $s_{\text {tot }}=3.6$. 
efficiency, $L_{\mathrm{MOT}}^{\mathrm{SB}} / \Phi_{\mathrm{SB}}=48(8) \%$, with a MOT loading rate slightly larger than that of a ZS-enhanced $\mathrm{Sr} 2 \mathrm{D}$ MOT source [21], and less than a factor of 10 lower than that of more-complex and more-power-demanding high-flux source systems based, for instance, on a combination of a Zeeamn slower, a 2D MOT, and deflection [43].

\section{B. Kinetic properties of the sideband-enhanced 2D MOT}

The kinetic properties of a $\mathrm{Sr}$ cold atomic beam generated by a 2D MOT have been studied recently [21], in particular as a function of the push-beam and 2D MOTbeam intensities. We verify these findings in our setup and extend the study to the addition of the sideband beam.

The longitudinal velocity is measured by the time-offlight technique. A push-beam pulse of $5 \mathrm{~ms}$ accelerates the 2D MOT atoms toward the science cell. The longitudinalvelocity distribution is estimated by our recording the fluorescence time distribution $f(t)$ as measured at the MOT center. We compute the longitudinal-velocity distribution as $f(v)=f(d / t)$, where $d=36.5(5) \mathrm{cm}$ is the 2D MOTto-MOT distance. Compared with the single-frequency 2D MOT, we do not observe any change in peak velocity or in velocity dispersion. The peak velocity $v_{L}$ for the optimal push saturation parameter $s_{\text {push }}=0.34$ is $22.5 \mathrm{~m} / \mathrm{s}$.

The atomic beam transverse velocity is measured by Doppler spectroscopy with and without our powering the sideband beam. The transverse velocity is extracted from the Doppler profile by our fixing the Lorentzian component due to the natural linewidth of the ${ }^{1} S_{0}{ }^{1} P_{1}$ probe transition and the saturation broadening $\left(s_{\text {probe }}=0.1\right.$ ). The measurement results are shown in Fig. 9, yielding Doppler broadening $\sigma_{2 \mathrm{D}}(T)=3.6(8) \mathrm{MHz}$ and $\sigma_{\mathrm{SB}}(T)=0.8(3.0) \mathrm{MHz}$, respectively. This corresponds to a transverse temperature of $14(7) \mathrm{mK}$ for the 2D MOT and 0.7(5.0) $\mathrm{mK}$ for the sideband-enhanced 2D MOT, as shown in the inset in Fig. 9. Compared with the Doppler temperature at $s_{2 \mathrm{D}}=6.6$ and $\Delta_{2 \mathrm{D}}=-1.6 \Gamma$, which is $2.1 \mathrm{mK}$, the $2 \mathrm{D}$ MOT result is nearly 7 times higher, while the sidebandenhanced case shows an upper-limit temperature almost 3 times higher. We also estimate the transverse temperature of the atomic beam resulting from $\mathrm{MC}$ numerical simulations, which presents transverse temperatures of 2.9(1) and 2.12(4) $\mathrm{mK}$, respectively. While the MC results confirm a colder beam for the sideband-enhanced case, they still miss the extra heating effects that can be explained by transverse spatial intensity fluctuations of the optical molasses in the 2D MOT [44].

From the measured transverse and longitudinal velocities, we derive atomic beam divergences $\theta_{2 \mathrm{D}} \equiv v_{t} / v_{L}=$ $75(17) \mathrm{mrad}$ and $\theta_{\mathrm{SB}} \leq 58 \mathrm{mrad}$. Finally, from the beam divergences and the atomic fluxes, we estimate the atomic beam radiant intensity, or sometimes called the beam "brightness," $\mathcal{J} \equiv \Phi /\left(\pi \theta^{2}\right)$. For the single-frequency

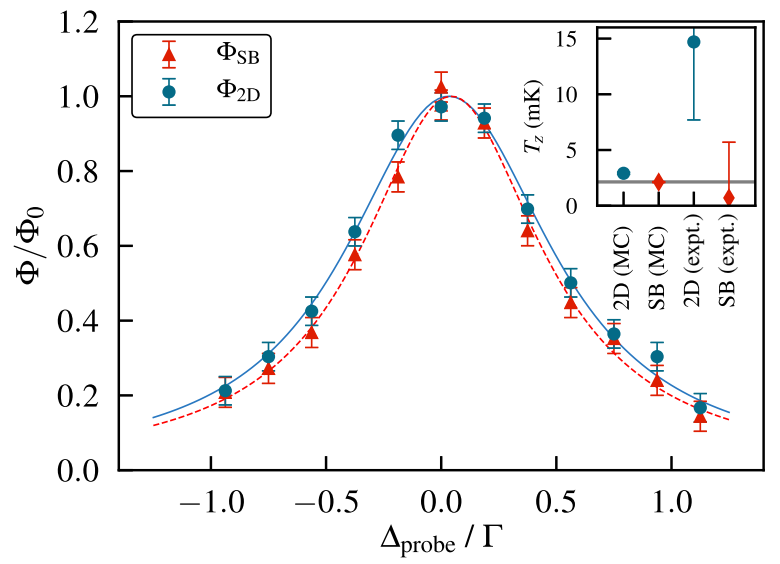

FIG. 9. Doppler spectrum from transverse spectroscopy on the 2D MOT Sr atomic beam. The inset shows the corresponding Doppler temperatures compared with the $\mathrm{MC}$ simulation results and the corresponding Doppler limit.

2D MOT we obtained brightness

$$
\mathcal{J}_{2 \mathrm{D}}=3.5(8) \times 10^{10} \text { atoms } / \mathrm{s} \mathrm{sr},
$$

while for the sideband-enhanced beam, the brightness

$$
\mathcal{J}_{\mathrm{SB}} \geq 1.4 \times 10^{11} \text { atoms } / \mathrm{s} \mathrm{sr} \text {. }
$$

This results represent a factor-of-4 improvement with respect to the single-frequency $2 \mathrm{D}$ MOT, making sideband enhancement a promising technique for optimal transfer to $2 \mathrm{D}$ optical molasses working on a narrow-linewidth intercombination transition of strontium for continuous Bose-Einstein condensate production and continuousoptical-clock proposals [45].

\section{Comparison with the Zeeman-slower enhancement}

An alternative method to increase the 2D MOT capture rate is to direct another slowing beam toward the hot atomic beam generated by the oven, which, exploiting the decreasing tail of the 2D MOT magnetic field, can efficiently scatter faster atoms along the beam direction similarly to a ZS. This approach was previously demonstrated in similar setups $[18,20,46]$.

We use the beam generated by the sideband AOM as a Zeeman slowing beam, shaped to have beam width $w_{Z S}=$ $6 \mathrm{~mm}$. We partially scan over the ZS parameters, which results in a maximum number of atoms in the MOT $N_{\mathrm{ZS}}$ of $3.8(1) \times 10^{6}$, obtained with $\Delta_{\mathrm{ZS}}=-8.1 \Gamma$ and $P_{\mathrm{ZS}}=$ $140 \mathrm{~mW}$, while we keep $P_{2 \mathrm{D}}=36 \mathrm{~mW}$ and $\Delta_{2 \mathrm{D}}=-1.5 \Gamma$ fixed. Blocking the ZS beam, we observe a gain in the atomic number on the order of 4 , in agreement with the experimental observation in Ref. [21].

Because of the short distance (about $25 \mathrm{~cm}$ ) between the oven aperture and the optical window facing it to send 
the ZS beam, we heat the window flange up to $350{ }^{\circ} \mathrm{C}$ to prevent metallization. However, we first observe a fast degradation of the atomic source flux, soon followed by the full metallization of the window. This prevents us from performing a fine optimization of $s_{\mathrm{ZS}}$ and $\Delta_{\mathrm{ZS}}$ as shown in Fig. 8 and also from producing a stable MOT during the day.

A possible way to reduce Sr metallization of the ZS window is to increase the distance between the oven and the window itself by means of a vacuum extension (at least a 1-m tube with smaller diameter). However, this solution would compromise the compactness of the atomic source conceived by a 2D MOT, making the system more complex, more power consuming, and perhaps needing extra water cooling to avoid thermal stress to the vacuum system. To avoid the thermal stress due to a heated $\mathrm{ZS}$ window, a $45^{\circ}$ metallic mirror can be placed in the vacuum inside an elbow vacuum connection, avoiding direct exposure to the optical window. However, reflectivity degradation will still occur for a Sr beam, making this option not a permanent solution [47].

Another drawback of the ZS method is the fact that the quantization axis of the magnetic field along the hotatom direction means that only half of the linear-polarized ZS light power has the correct circular polarization. This means that at least half of the ZS optical power is wasted in the slowing process. In contrast, the sideband beams have a well-defined polarization in the capture region, so all the power used is effective in the cooling and trapping process.

\section{PROSPECTS FOR OPTICAL CLOCKS}

\section{A. Application of sideband enhancement to other alkaline-earth atoms}

It is interesting to extend the discussion of the sidebandenhancement method to other atomic species, in particular those used in optical clocks. We use MC simulation to investigate the potential trapping performances of additional atomic species. Table I shows the main optical and atomic parameters for alkaline-earth and alkaline-earthlike atomic species currently in use in optical clock experiments. In particular, we consider the broad ${ }^{1} S_{0}{ }^{1} P_{1}$ strong dipole transition as the cooling transition, fixing the atomic vapor pressure at $0.1 \mathrm{~Pa}$ for all the species. We run our MC simulation with these parameters, with a total available saturation parameter $s_{\text {tot }}=6.56$, the same magnetic field gradient, and the same laser-beam widths as for our previously described apparatus.

The simulation workflow is as follows: first we simulate the single-frequency 2D MOT, looking for the optimal detuning $\Delta_{2 \mathrm{D}}$ at half of $s_{\text {tot }}$; then we add the sideband at $\Delta_{\mathrm{SB}}=2 \Delta_{2 \mathrm{D}}$, which is basically the result we found in Sec. VI for $\mathrm{Sr}$, and we scan the sideband-enhanced 2D MOT at different sideband saturation-parameter values $s_{\mathrm{SB}}$. Because of the extremely high saturation intensities of
$\mathrm{Cd}$ and $\mathrm{Hg}$, which makes unrealistic the application of this method, they are excluded from this numerical study.

The MC simulation results are reported in Table I. Here we clearly see that the sideband enhancement is more effective for those atoms having a lower maximum acceleration $a_{\max }$. This dependence can be understood by looking at the definition of the maximum capture velocity in Eq. (2). It can be achieved only for a light field uniformly resonant with the atomic transition and fully saturated all along the trap diameter. This means that the broader the cooling-transition linewidth $\Gamma$ is (and thus the higher $\left.a_{\max }\right)$, the closer the MOT is to its capture limit, implying that the expected enhancement factor is lower. Furthermore, according to Eq. (1), we would expect the sideband enhancement to work better for light species, in particular where $v_{\text {th }}$ is higher and the quartic dependence of the loading rate on the capture velocity is a more-accurate approximation. Hence, we can work out a sideband-enhancement-factor functional dependence:

$$
\eta(X) \propto \frac{v_{\mathrm{th}}(X)^{2}}{a_{\max }(X)}
$$

where $X$ is the atomic species considered. By accident, $\mathrm{Sr}$, $\mathrm{Ca}$, and $\mathrm{Yb}$ have very similar $v_{\mathrm{th}}(X)^{2} / a_{\max }(X)$ values, and the resulting $\eta$ is the same within the numerical error.

We also report in Table I the absolute capture efficiency for the sideband-enhanced 2D MOT atomic source $r(X)$. MC simulations show that the highest capture rate is predicted for $\mathrm{Yb}$, followed by $\mathrm{Sr}$, two of the strongest candidates for a possible redefinition of the second on the basis of optical atomic clocks [48].

\section{B. Metrological advantages in optical clocks}

Finally, we outline the expected accuracy impact of our 2D MOT atomic source when applied to an optical lattice clock. Three sources of systematic effects can be significantly suppressed by our atomic source. The first effect is the Zeeman shift due to stray magnetic fields from magnets. In our apparatus, the 2D MOT magnets should exert a null magnetic field in the MOT center if they are symmetrically placed. Slight misplacements may result in a residual magnetic field on the atomic clock sample. Assuming a realistic misalignment on the order of $2 \mathrm{~mm}$, we expect a residual-magnetic-field amplitude on the order of $2 \times 10^{-7} \mathrm{~T}$, which is much lower than typical magnetic biases in a laboratory. This corresponds to a linear Zeeman shift of about $200 \mathrm{mHz}$ and a relative quadratic Zeeman shift of about $2 \times 10^{-21}$ for the ${ }^{87} \mathrm{Sr}$ clock transition. Also the expected field gradient is about $3 \times 10^{-6} \mathrm{~T} / \mathrm{m}$, which yields a negligible uncertainty on a $1-\mathrm{mm}$ atomic cloud.

The second major advantage of this system is the suppression of direct BBR from the hot oven to the atoms. The residual contribution of the hot oven to the BBR shift is due to the induced heating of the remaining vacuum system, 
TABLE I. Sideband enhancement for alkaline-earth and alkaline-earth-like atomic species. On the left side of the table the mostrelevant parameters for cooling and trapping atoms on the ${ }^{1} S_{0}{ }^{-1} P_{1}$ strong dipole transition are shown, whereas we estimate the thermal and kinetic properties of every atomic species at pressure $p_{0}=0.1 \mathrm{~Pa}$. On the right, MC optimization results for the $2 \mathrm{D}$ MOT detuning $\Delta_{2 \mathrm{D}}$, the fraction of trapped atoms, and the enhancement factor $\eta$ for each alkaline-earth species are given.

\begin{tabular}{|c|c|c|c|c|c|c|c|c|c|}
\hline \multirow{2}{*}{ Atom } & \multirow{2}{*}{$\begin{array}{c}\lambda \\
(\mathrm{nm})\end{array}$} & \multirow{2}{*}{$\begin{array}{c}\Gamma / 2 \pi \\
(\mathrm{MHz}) \\
\end{array}$} & \multirow{2}{*}{$\begin{array}{c}I_{\mathrm{sat}} \\
\left(\mathrm{mW} / \mathrm{cm}^{2}\right)\end{array}$} & \multirow{2}{*}{$\begin{array}{c}a_{\max } \\
\left(10^{6} \mathrm{~m} / \mathrm{s}^{2}\right)\end{array}$} & \multirow{2}{*}{$\begin{array}{c}T\left(p_{0}\right) \\
(\mathrm{K}) \\
\end{array}$} & \multirow{2}{*}{$\begin{array}{c}v_{\mathrm{th}}\left(p_{0}\right) \\
(\mathrm{m} / \mathrm{s})\end{array}$} & $\Delta_{2 \mathrm{D}} / \Gamma$ & $r^{\mathrm{MC}} f_{\text {cut }}$ & $\eta$ \\
\hline & & & & & & & \multicolumn{3}{|c|}{ (ppm) } \\
\hline${ }^{24} \mathrm{Mg}$ & 285.30 & 80.95 & 455 & 14.8 & 641 & 679 & -2.28 & 77 & 1.0 \\
\hline${ }^{40} \mathrm{Ca}$ & 422.79 & 34.63 & 59.9 & 2.69 & 788 & 583 & -2.5 & 103 & 2.1 \\
\hline${ }^{88} \mathrm{Sr}$ & 460.86 & 31.99 & 42.7 & 1.01 & 725 & 379 & -1.76 & 260 & 2.1 \\
\hline${ }^{138} \mathrm{Ba}$ & 553.70 & 18.33 & 14.1 & 0.31 & 826 & 321 & -0.89 & 60 & 2.9 \\
\hline${ }^{114} \mathrm{Cd}$ & 228 & 91 & 1005 & 4.64 & 485 & 273 & & & \\
\hline${ }^{174} \mathrm{Yb}$ & 398.91 & 29 & 59.8 & 0.54 & 673 & 258 & -1.42 & 316 & 2.2 \\
\hline${ }^{198} \mathrm{Hg}$ & 185 & 120 & 2481 & 4.24 & 286 & 157 & & & \\
\hline
\end{tabular}

which results in a temperature inhomogeneity that must be accessed for high-accuracy clocks. Assuming that the high-vacuum region is warmed up to $40^{\circ} \mathrm{C}$ and stainlesssteel emissivity $\varepsilon_{\mathrm{SS}}=0.27$, evaluated at $T_{\mathrm{HV}}$, the effective temperature seen by the atoms is [49]

$$
T_{\mathrm{eff}}^{4}=\frac{\Omega_{\mathrm{DPC}} \varepsilon_{\mathrm{SS}} T_{\mathrm{HV}}^{4}+\left(4 \pi-\Omega_{\mathrm{DPC}}\right) \varepsilon_{c} T_{c}^{4}}{\Omega_{\mathrm{DPC}} \varepsilon_{\mathrm{SS}}+\left(4 \pi-\Omega_{\mathrm{DPC}}\right) \varepsilon_{c}},
$$

where $\Omega_{\mathrm{DPC}}$ is the whole solid angle allowed by the DPC, while $\varepsilon_{c}$ and $T_{c}$ refer to the science chamber, which can have very-well-known and controlled BBR [13]. Expanding this expression with respect to the temperature difference $\Delta T=T_{\mathrm{HV}}-T_{c}$, we can calculate the temperature increase seen by the atoms as

$$
\Delta T_{\mathrm{eff}} \approx \frac{\Omega_{\mathrm{DPC}} \varepsilon_{\mathrm{SS}}}{\Omega_{\mathrm{DPC}} \varepsilon_{\mathrm{SS}}+\left(4 \pi-\Omega_{\mathrm{DPC}}\right) \varepsilon_{c}} \Delta T=2.2 \times 10^{-6} \mathrm{~K} .
$$

This contributes to the BBR shift an amount on the order of $1 \times 10^{-20}$ in relative units for the Sr clock transition, far below the $10^{-18}$ level. We stress that this estimate is a worst-case scenario.

The third suppressed systematic effect is the collisional shift from the atomic beam. The 2D MOT atomic source naturally provides an "optical atomic shutter" with infinite extinction ratio once both the cooling beam and the push beam are switched off. It also avoids the vibrations of an in-vacuum mechanical shutter that can introduce Doppler shifts during interrogation of atoms.

\section{CONCLUSIONS}

In this work we demonstrate and fully characterize a robust method to enhance the atomic flux generated by a Sr 2D MOT by adding a second frequency to the 2D MOT beams. The experimental implementation of the sidebandenhancement method requires only a simple optical setup and a proper alignment of the sideband beam with the main 2D MOT beam. The resulting bright atomic source can deliver more than $1.4 \times 10^{11}$ atoms/s sr if the total available power for the atomic source is $200 \mathrm{~mW}$. This cold atomic flux can be efficiently loaded in a 3D MOT for ultracold-atom experiments, preventing direct sight of the hot atomic oven and providing an efficient optical shutter for the atomic beam. This result represents an enhancement in MOT loading by a factor of 2.3 with respect to a single-frequency 2D MOT-based atomic source.

A dedicated Monte Carlo simulation, which well predicts the experimental data for our $\mathrm{Sr}$ atomic source, shows that this technique is a valid method to increase the number of atomic sources based on other alkaline-earth species and alkaline-earth-like species, such as $\mathrm{Ca}$ and $\mathrm{Yb}$, paving the way for compact atomic sources suitable for transportable optical clocks or optical-clock-transition-based gravimeters [50,51].

\section{ACKNOWLEDGMENTS}

We thank U. Sterr for inspirational discussions about the sideband-enhanced MOT, D. Racca, E. Bertacco, M. Bertinetti, and A. Barbone for laboratory assistance, and F. Bregolin for critical reading of the manuscript. We acknowledge funding of the project EMPIR-USOQS; EMPIR projects are co-funded by the European Union's Horizon 2020 research and innovation program and the EMPIR participating states. We also acknowledge the QuantERA project Q-Clocks, ASI, and Provincia Autonoma di Trento for financial support.

[1] Alexander D. Cronin, Jörg Schmiedmayer, and David E. Pritchard, Optics and interferometry with atoms and molecules, Rev. Mod. Phys. 81, 1051 (2009).

[2] I. M. Georgescu, S. Ashhab, and Franco Nori, Quantum simulation, Rev. Mod. Phys. 86, 153 (2014).

[3] Andrew D. Ludlow, Martin M. Boyd, Jun Ye, E. Peik, and P. O. Schmidt, Optical atomic clocks, Rev. Mod. Phys. 87, 637 (2015). 
[4] G. Wilpers, C. W. Oates, S. A. Diddams, A. Bartels, T. M. Fortier, W. H. Oskay, J. C. Bergquist, S. R. Jefferts, T. P. Heavner, T. E. Parker, and L. Hollberg, Absolute frequency measurement of the neutral ${ }^{40} \mathrm{Ca}$ optical frequency standard at $657 \mathrm{~nm}$ based on microkelvin atoms, Metrologia 44, 146 (2007).

[5] Ichiro Ushijima, Masao Takamoto, Manoj Das, Takuya Ohkubo, and Hidetoshi Katori, Cryogenic optical lattice clocks, Nat. Photonics 9, 185 (2015).

[6] A. P. Kulosa, D. Fim, K. H. Zipfel, S. Rühmann, S. Sauer, N. Jha, K. Gibble, W. Ertmer, E. M. Rasel, M. S. Safronova, U. I. Safronova, and S. G. Porsev, Towards a Mg Lattice Clock: Observation of the ${ }^{1} S_{0}-{ }^{3} P_{0}$ Transition and Determination of the Magic Wavelength, Phys. Rev. Lett. 115, 240801 (2015).

[7] W. F. McGrew, X. Zhang, R. J. Fasano, S. A. Schäffer, K. Beloy, D. Nicolodi, R. C. Brown, N. Hinkley, G. Milani, M. Schioppo, T. H. Yoon, and A. D. Ludlow, Atomic clock performance enabling geodesy below the centimetre level, Nature 564, 87 (2018).

[8] N. Poli, M. Schioppo, S. Vogt, St. Falke, U. Sterr, Ch. Lisdat, and G. M. Tino, A transportable strontium optical lattice clock, Appl. Phys. B 117, 1107 (2014).

[9] AOSense Inc., Permanent magnet axial field Zeeman slower, U.S. Patent No. 8,710,428 B1 (2014).

[10] C. J. Dedman, J. Nes, T. M. Hanna, R. G. Dall, K. G. H. Baldwin, and A. G. Truscott, Optimum design and construction of a zeeman slower for use with a magneto-optic trap, Rev. Sci. Instrum. 75, 5136 (2004).

[11] S. Origlia, M. S. Pramod, S. Schiller, Y. Singh, K. Bongs, R. Schwarz, A. Al-Masoudi, S. Dörscher, S. Herbers, S. Häfner, U. Sterr, and Ch. Lisdat, Towards an optical clock for space: Compact, high-performance optical lattice clock based on bosonic atoms, Phys. Rev. A 98, 053443 (2018).

[12] Kurt Gibble, Scattering of Cold-Atom Coherences by Hot Atoms: Frequency Shifts from Background-Gas Collisions, Phys. Rev. Lett. 110, 180802 (2013).

[13] K. Beloy, N. Hinkley, N. B. Phillips, J. A. Sherman, M. Schioppo, J. Lehman, A. Feldman, L. M. Hanssen, C. W. Oates, and A. D. Ludlow, Atomic Clock with $1 \times 10^{-18}$ Room-Temperature Blackbody Stark Uncertainty, Phys. Rev. Lett. 113, 260801 (2014).

[14] K. Dieckmann, R. J. C. Spreeuw, M. Weidemüller, and J. T. M. Walraven, Two-dimensional magneto-optical trap as a source of slow atoms, Phys. Rev. A 58, 3891 (1998).

[15] J. Schoser, A. Batär, R. Löw, V. Schweikhard, A. Grabowski, Yu. B. Ovchinnikov, and T. Pfau, Intense source of cold $\mathrm{rb}$ atoms from a pure two-dimensional magneto-optical trap, Phys. Rev. A 66, 023410 (2002).

[16] A. Witte, Th. Kisters, F. Riehle, and J. Helmcke, Laser cooling and deflection of a calcium atomic beam, J. Opt. Soc. Am. B 9, 1030 (1992).

[17] T. G. Tiecke, S. D. Gensemer, A. Ludewig, and J. T. M. Walraven, High-flux two-dimensional magneto-opticaltrap source for cold lithium atoms, Phys. Rev. A 80, 013409 (2009).

[18] G. Lamporesi, S. Donadello, S. Serafini, and G. Ferrari, Compact high-flux source of cold sodium atoms, Rev. Sci. Instrum. 84, 063102 (2013).
[19] V. D. Vaidya, J. Tiamsuphat, S. L. Rolston, and J. V. Porto, Degenerate bose-fermi mixtures of rubidium and ytterbium, Phys. Rev. A 92, 043604 (2015).

[20] G. Colzi, E. Fava, M. Barbiero, C. Mordini, G. Lamporesi, and G. Ferrari, Production of large bose-einstein condensates in a magnetic-shield-compatible hybrid trap, Phys. Rev. A 97, 053625 (2018).

[21] Ingo Nosske, Luc Couturier, Fachao Hu, Canzhu Tan, Chang Qiao, Jan Blume, Y. H. Jiang, Peng Chen, and Matthias Weidemüller, Two-dimensional magneto-optical trap as a source for cold strontium atoms, Phys. Rev. A 96, 053415 (2017).

[22] Xinye Xu, Thomas H. Loftus, John L. Hall, Alan Gallagher, and Jun Ye, Cooling and trapping of atomic strontium, J. Opt. Soc. Am. B 20, 968 (2003).

[23] C. Monroe, W. Swann, H. Robinson, and C. Wieman, Very Cold Trapped Atoms in a Vapor Cell, Phys. Rev. Lett. 65, 1571 (1990).

[24] Götz Zinner, Ph.D. thesis, University of Hannover, 1998.

[25] J. Hoffnagle, Proposal for continuous white-light cooling of an atomic beam, Opt. Lett. 13, 102 (1988).

[26] M. Zhu, C. W. Oates, and J. L. Hall, Continuous HighFlux Monovelocity Atomic Beam Based on a Broadband Laser-Cooling Technique, Phys. Rev. Lett. 67, 46 (1991).

[27] Brian P. Anderson and Mark A. Kasevich, Enhanced loading of a magneto-optic trap from an atomic beam, Phys. Rev. A 50, R3581 (1994).

[28] Jae Hoon Lee and Jongchul Mun, Optimized atomic flux from a frequency-modulated two-dimensional magnetooptical trap for cold fermionic potassium atoms, J. Opt. Soc. Am. B 34, 1415 (2017).

[29] F. Riehle, H. Schnatz, B. Lipphardt, G. Zinner, T. Trebst, T. Binnewies, G. Wilpers, and J. Helmcke, in Proceedings of the 1999 Joint Meeting of the European Frequency and Time Forum and the IEEE International Frequency Control Symposium (IEEE, Besancon, France, 1999), Vol. 2, p. 700 .

[30] M. G. Tarallo, D. Calonico, F. Levi, M. Barbiero, G. Lamporesi, and G. Ferrari, in 2017 Joint Conference of the European Frequency and Time Forum and IEEE International Frequency Control Symposium (EFTF/IFC) (IEEE, Besancon, France, 2017).

[31] C. B. Alcock, V. P. Itkin, and M. K. Horrigan, Vapour pressure equations for the metallic elements: 298-2500 K, Can. Metall. Q. 23, 309 (1984).

[32] J. A. Giordmaine and T. C. Wang, Molecular beam formation by long parallel tubes, J. Appl. Phys. 31, 463 (1960).

[33] N. Poli, G. Ferrari, M. Prevedelli, F. Sorrentino, R. E. Drullinger, and G. M. Tino, Laser sources for precision spectroscopy on atomic strontium, Spectrochim. Acta, Part A: Mol. Biomol. Spectrosc. 63, 981 (2006).

[34] W. Wohlleben, F. Chevy, K. Madison, and J. Dalibard, An atom faucet, Eur. Phys. J. D 15, 237 (2001).

[35] James M. Kohel, Jaime Ramirez-Serrano, Robert J. Thompson, Lute Maleki, Joshua L. Bliss, and Kenneth G. Libbrecht, Generation of an intense cold-atom beam from a pyramidal magneto-optical trap: Experiment and simulation, J. Opt. Soc. Am. B 20, 1161 (2003). 
[36] Saptarishi Chaudhuri, Sanjukta Roy, and C. S. Unnikrishnan, Realization of an intense cold $\mathrm{Rb}$ atomic beam based on a two-dimensional magneto-optical trap: Experiments and comparison with simulations, Phys. Rev. A 74, 023406 (2006).

[37] Asaf Szulc, Master's thesis, Ben-Gurion University of the Negev, 2016.

[38] Ryan K. Hanley, Paul Huillery, Niamh C. Keegan, Alistair D. Bounds, Danielle Boddy, Riccardo Faoro, and Matthew P. A. Jones, Quantitative simulation of a magneto-optical trap operating near the photon recoil limit, J. Mod. Opt. 65, 667 (2017).

[39] R. W. Mu, Z. L. Wang, Y. L. Li, X. M. Ji, and J. P. Yin, A controllable double-well optical trap for cold atoms (or molecules) using a binary $\pi$-phase plate: Experimental demonstration and monte carlo simulation, Eur. Phys. J. D 59, 291 (2010).

[40] Daniel Comparat, Molecular cooling via sisyphus processes, Phys. Rev. A 89, 043410 (2014).

[41] A. D. Bounds, N. C. Jackson, R. K. Hanley, R. Faoro, E. M. Bridge, P. Huillery, and M. P. A. Jones, RydbergDressed Magneto-Optical Trap, Phys. Rev. Lett. 120, 183401 (2018).

[42] W. H. Enright, The numerical analysis of ordinary differential equations: Runge-Kutta and general linear methods (J. C. Butcher), SIAM Rev. 31, 693 (1989).

[43] Tao Yang, Kanhaiya Pandey, Mysore Srinivas Pramod, Frederic Leroux, Chang Chi Kwong, Elnur Hajiyev, Zhong Yi Chia, Bess Fang, and David Wilkowski, A high flux source of cold strontium atoms, Eur. Phys. J. D 69, 226 (2015).

[44] Thierry Chanelière, Jean-Louis Meunier, Robin Kaiser, Christian Miniatura, and David Wilkowski, Extra-heating mechanism in doppler cooling experiments, J. Opt. Soc. Am. B 22, 1819 (2005).

[45] Shayne Bennetts, Chun-Chia Chen, Benjamin Pasquiou, and Florian Schreck, Steady-State Magneto-optical Trap with 100-Fold Improved Phase-Space Density, Phys. Rev. Lett. 119, 223202 (2017).

[46] Ingo Nosske, Ph.D. dissertation, University of Science and Technology of China, 2018.

[47] J. Huckans, W. Dubosclard, E. Maréchal, O. Gorceix, B. Laburthe-Tolra, and M. Robert-de-Saint-Vincent, Note on the reflectance of mirrors exposed to a strontium beam, arXiv:1802.08499 (2018).

[48] Fritz Riehle, Towards a redefinition of the second based on optical atomic clocks, C.R. Phys. 16, 506 (2015).

[49] T. Middelmann, C. Lisdat, S. Falke, J. S. R. Vellore Winfred, F. Riehle, and U. Sterr, Tackling the blackbody shift in a strontium optical lattice clock, IEEE Trans. Instrum. Meas. 60, 2550 (2011).

[50] Liang $\mathrm{Hu}$, Nicola Poli, Leonardo Salvi, and Guglielmo M. Tino, Atom Interferometry with the Sr Optical Clock Transition, Phys. Rev. Lett. 119, 263601 (2017).

[51] Tomoya Akatsuka, Tadahiro Takahashi, and Hidetoshi Katori, Optically guided atom interferometer tuned to magic wavelength, Appl. Phys Express 10, 112501 (2017). 